\title{
Three-Dimensional Texture
} Analysis after Bunge and Roe: Correspondence Between the Respective Mathematical Techniques

\author{
C. ESLING, E. BECHLER-FERRY and H. J. BUNGE
}

Laboratoire de Métallurgie Structurale, Faculté des Sciences, Ile du Saulcy, F-57045 Metz Cedex-France Institut für Metallkunde und Metallphysik, D-3392 Clausthal-Zellerfeld, F.R.G.

(Received November 13, 1981)

Dedicated to Prof. P. Paulitsch for his 60th birthday.

Bunge's and Roe's three-dimensional texture analysis methods, although both founded on harmonic analysis, show some differences between the various mathematical techniques used.

This paper establishes the correspondence relation between the respective mathematical techniques allowing one to compare works done in either variant. Taking the latest developments in three dimensional texture analysis into account, the correspondence relations hold for the odd degrees $l$ as well as for the even ones.

Finally numerical tables give the extension of the symmetry coefficients $\dot{B}_{l}^{4 m \mu}$ (after Bunge) and $R_{4 n \mu}^{l}$ (after Roe) to all the degrees $l$ of the series expansion, even and odd, including $l=34$.

\section{INTRODUCTION}

In 1965, Bunge on the one hand and Roe (1965) on the other hand have simultaneously published a theory about three-dimensional analysis of crystallographic textures. Their theories are founded on the same principle: using pole figures which are bi-dimensional projections for the purpose of calculating the three-dimensional texture function $f(g)$. Although the base of their methods is similar, the formalism is slightly different, essentially because of:

- different definition of the Euler angles

- the utilization of polynomials having different phase- and normconventions 
-the way of taking the symmetries into account.

As the scientific publications in the field of texture analysis use either terminologies, the comparison between the various results published requires some correlation relations that we intend to establish.

\section{THE EULER ANGLES}

\section{Definition of the Euler angles after Bunge and Roe}

The texture function $f(g)$ describes the orientation distribution of the crystallites in the sample. These orientations are generally described by means of Euler angles making the coinciding of two orthogonal reference systems possible:

$K_{A}$ bound to the sample and $K_{B}$ bound to the crystallographic lattice of grains.

This coincidence is generally achieved by three successive rotations around the $K_{B}$ reference axes.
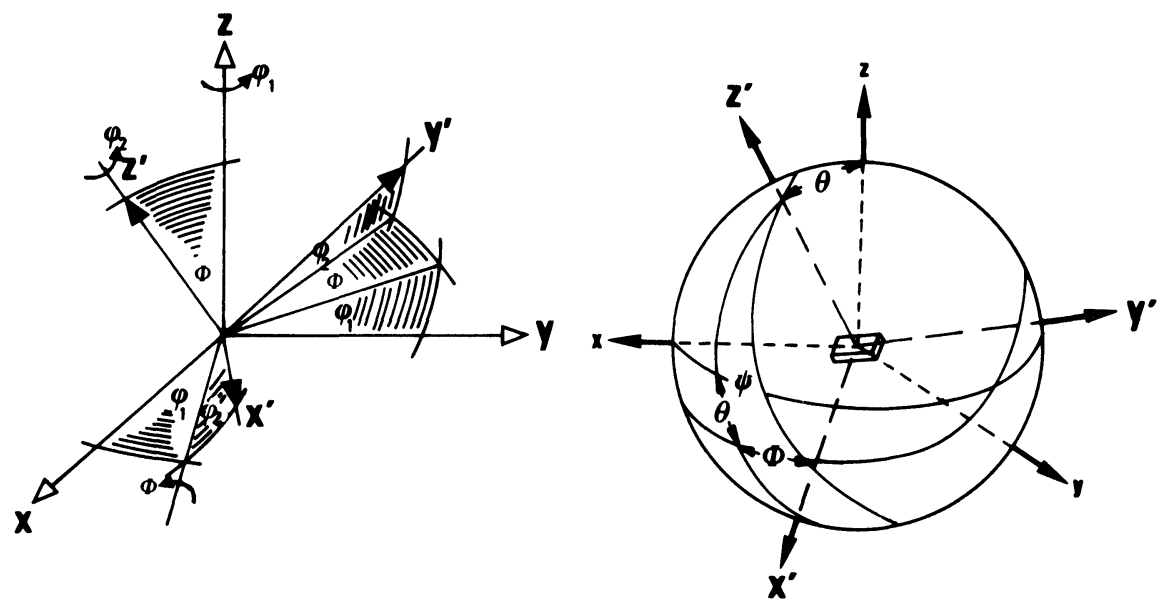

FIGURE 1a Euler angles after Bunge (1969). FIGURE 1b Euler angles after Roe (1965).

Bunge uses the triplet $\left\{\varphi_{1}, \Phi, \varphi_{2}\right\}$ corresponding to (Figure 1a):

- a rotation $\varphi_{1}$ around $Z^{\prime}$

- a rotation $\Phi$ around $X^{\prime}$ (in the new position)

- a rotation $\varphi_{2}$ around $Z^{\prime}$ (in the new position)
The angles $\{\psi, \theta, \varphi\}$ used by Roe correspond to Figure $1 \mathrm{~b}$ ):

-a rotation $\psi$ around $Z^{\prime}$

-a rotation $\theta$ around $Y^{\prime}$ (in the new position)

-a rotation $\varphi$ around $Z^{\prime}$ (in the new position) 
The variables are different only because of the second rotation, whose axes $\left(X^{\prime}\right.$ and $\left.Y^{\prime}\right)$ are orthogonal, which introduces a phase difference of amplitude $\frac{\Pi}{2}$. Thus, both triplets described the same orientation of the $K_{B}$ reference if they are linked by the relations:

$$
\begin{aligned}
\varphi_{1} & =\psi+\frac{\pi}{2} \\
\Phi & =\theta \\
\varphi_{2} & =\varphi-\frac{\pi}{2}
\end{aligned}
$$

\section{The invariant measure $d g$}

The O.D.F. depends on the Euler angles:

$$
f(g)=f\left(\varphi_{1}, \Phi, \varphi_{2}\right)
$$

This density function has to be positive and to verify the normalization condition:

$$
\int_{\Omega} f(g) \cdot d g=1
$$

The integral is extended over the whole of the Euler space:

$$
\begin{aligned}
& 0 \leqslant \varphi_{1} \leqslant 2 \pi \\
& 0 \leqslant \Phi \leqslant \pi \\
& 0 \leqslant \varphi_{2} \leqslant 2 \pi
\end{aligned}
$$

The Euler angles $\varphi_{1}, \Phi, \varphi_{2}$ constitute a system of curvilinear coordinates. In this system the differential integration element reads:

$$
d g=I\left(\varphi_{1}, \Phi, \varphi_{2}\right) \cdot d \varphi_{1} \cdot d \Phi \cdot d \varphi_{2} .
$$

It should be noticed that this differential volume element is not the simple product of the differentials of each variable, as in the case of Cartesian coordinates, for which:

$$
d v=d x \cdot d y \cdot d z
$$

The differential integration element (also named "invariant measure") used by Bunge:

$$
d g=\frac{1}{8 \pi^{2}} \sin \Phi \cdot d \Phi \cdot d \varphi_{1} \cdot d \varphi_{2}
$$


is such as:

$$
\int_{0}^{2 \pi} \int_{0}^{\pi} \int_{0}^{2 \pi} \frac{1}{8 \pi^{2}} \sin \Phi \cdot d \Phi \cdot d \varphi_{1} \cdot d \varphi_{2}=1
$$

It differs from the differential element used by Roe by the factor $\frac{1}{8 \pi^{2}}$ :

$$
\begin{gathered}
\int_{0}^{2 \pi} \int_{-1}^{+1} \int_{0}^{2 \pi} d \psi \cdot d \xi \cdot d \varphi=8 \pi^{2} \\
\text { with } \xi=\cos \theta
\end{gathered}
$$

This selfsame factor is consequently found again between the functions of orientation density:

$$
w(\psi, \theta, \varphi)=\frac{1}{8 \pi^{2}} f\left(\varphi_{1}, \Phi, \varphi_{2}\right)
$$

used by Bunge and Roe respectively.

\section{THE THREE DIMENSIONAL FUNCTIONS}

\section{Relation between the polynomials $P_{I}^{m n}(\Phi)$ and $\boldsymbol{Z}_{\boldsymbol{I m n}}(\xi)$}

A convenient representation of the texture function $f(g)$ is its development into series on the basis of the generalized spherical harmonics. According to H. J. Bunge, this development reads:

$$
f(g)=\sum_{l} \sum_{m} \sum_{n} C_{l}^{m n} T_{l}^{m n}(g)
$$

or

$$
f\left(\varphi_{1}, \Phi, \varphi_{2}\right)=\sum_{l} \sum_{m} \sum_{n} C_{l}^{m n} e^{i m \varphi_{2}} P_{l}^{m n}(\Phi) e^{i n \varphi_{1}}
$$

In R. J. Roe's terminology the corresponding development reads:

$$
w(\psi, \xi, \varphi)=\sum_{l} \sum_{m} \sum_{n} W_{l m n} e^{-i m \varphi} Z_{l m n}(\xi) \cdot e^{-i n \varphi}
$$

The expression of the generalized spherical harmonics contains polynomials which are associated to Jacobi's polynomials.

$$
P_{l}^{m n}(\cos \Phi)=P_{l}^{m n}(x)=\frac{(-1)^{l-m_{i} n-m}}{2^{l}(l-m) !}\left[\frac{(l-m) !(l+n) !}{(l+m) !(l-n) !}\right]^{1 / 2} \times
$$




$$
\times(1-x)^{-\frac{n-m}{2}}(1+x)^{-\frac{n+m}{2}} \frac{d^{l-n}}{d x^{l-n}}\left[(1-x)^{l-m}(1+x)^{l+m}\right] .
$$

The $Z_{l m n}(\xi)$ polynomials only differ from the previous ones by a normalization factor:

$$
Z_{l m n}(\cos \Phi)=i^{n-m} \sqrt{\frac{2 l+1}{2}} P_{l}^{m n}(\cos \Phi)
$$

\section{Respective norms of the O.D.F.s}

The generalized spherical harmonics form a complete orthonormal basis for the functions which depend on the Euler angles:

$$
\int T_{l}^{m n}(g) \cdot T_{l^{\prime}}^{* m^{\prime} n^{\prime}}(g) \cdot d g=\frac{1}{2 l+1} \delta_{l l^{\prime}} \delta_{m n^{\prime}} \delta_{n n^{\prime}}
$$

With Roe's notations, these relations of orthonormalization read:

$$
\int e^{-i m \psi} Z_{l m n}(\xi) e^{-i n \varphi} \cdot e^{i m^{\prime} \psi} Z_{l^{\prime} m^{\prime} n^{\prime}}(\xi) e^{i n^{\prime} \varphi} d \psi \cdot d \xi \cdot d \varphi=4 \pi^{2} \delta_{l l^{\prime}} \delta_{m m^{\prime}} \delta_{n n^{\prime}}
$$

Let us recall that as the texture function $f(g)$ is a density function, it must verify the norm condition Eq. (3):

$$
\int w(\psi, \theta, \varphi) \sin \theta \cdot d \psi \cdot d \theta \cdot d \varphi=1
$$

The particular case in which the function considered corresponds to a perfect random orientation distribution gives:

$$
\left\{\begin{array}{l}
f(g)=f_{\text {rand }} \\
w(g)=w_{\text {rand }}
\end{array}\right.
$$

where $f_{\text {rand. }}$ and $w_{\text {rand. }}$ are two constants.

The above norm relations immediately lead to the norms of random distributions:

$$
\int_{\Omega} f_{\text {rand. }} \cdot d g=f_{\text {rand. }} \int_{\Omega} d g=1 \Rightarrow f_{\text {rand. }}=1
$$

and

$$
\int_{\Omega} w_{\text {rand. }} \sin \theta \cdot d \psi \cdot d \theta \cdot d \varphi=w_{\text {rand. }} \int_{\Omega} \sin \theta \cdot d \psi \cdot d \theta \cdot d \varphi=1 \Rightarrow w_{\text {rand. }}=\frac{1}{8 \pi^{2}}
$$

Imposing the norm condition to the serial development of $f(g)$ gives:

$$
\int\left(\sum C_{l}^{m n} T_{l}^{m n}(g)\right) d g=\sum C_{l}^{m n} \int T_{l}^{m n}(g)=1
$$


The integral of the generalized spherical harmonics may be considered as a particular case of the orthonormalization relations:

$$
\int T_{l}^{m n}(g) d g=\int T_{l}^{m n}(g) \cdot 1 \cdot d g=\int T_{l}^{m n}(g) \cdot T_{0}^{00}(g) \cdot d g=\delta_{l 0} \delta_{m 0} \delta_{n 0}
$$

Therefore the norm condition of $f(g)$ determines the value of the first coefficient of rank $l=0$ in the development:

$$
C_{0}^{00}=1
$$

and it follows from a similar calculation:

$$
W_{000}=\frac{\sqrt{2}}{8 \pi^{2}}
$$

Relation between the coefficients $C_{l}^{m n}$ and $W_{l}^{m n}$ of the O.D.F.s

The determination of the set of coefficients $\left\{C_{l}^{m n}\right\} l<l_{\max }$ of the $f(g)$ development constitutes an important stage in studying the texture of a polycrystal. Indeed, this set of coefficients contains the whole information about the orientation distribution in a strongly digested form, and enables the later computation of $f(g)$ as well as that of the anisotropic physical properties.

Starting from Bunge's development, let us replace the triplet $\left(\varphi_{1}, \Phi, \varphi_{2}\right)$ by $(\psi, \theta, \varphi)$ by applying the correspondence relations Eq. (1):

$$
f\left(\varphi_{1}, \Phi, \varphi_{2}\right)=\sum C_{l}^{m n} e^{i m\left(\varphi-\frac{\pi}{2}\right)} P_{l}^{m n}(\cos \theta) e^{i n\left(\psi+\frac{\pi}{2}\right)}
$$

Since $m$ and $n$ are dumb indices for summation, it is allowed to permute them and change their signs.

$$
f\left(\varphi_{1}, \Phi, \varphi_{2}\right)=\sum C_{l}^{-m-n} e^{-i n\left(\varphi-\frac{\pi}{2}\right)} P_{l}^{-n-m}(\cos \theta) e^{-i m\left(\psi+\frac{\pi}{2}\right)}
$$

Applying the symmetry relation of the polynomials $P_{l}^{m n}$ and Eq. (15)

$$
P_{l}^{-n-m}(\cos \theta)=P_{l}^{m n}(\cos \theta)=i^{m-n} \sqrt{\frac{2}{2 l+1}} Z_{l m n}(\cos \theta)
$$

we obtain the expression of spherical harmonics in Roe's terminology:

$$
f\left(\varphi_{1}, \Phi, \varphi_{2}\right)=\sum\left|\sqrt{\frac{2}{2 l+1} C_{l}^{-n-m} \mid}\right| e^{-i m \psi} Z_{l m n}(\cos \theta) e^{-i n \varphi} \mid
$$

Therefore the constants which multiply them are Roe's coefficients but for the norm factor $1 / 8 \pi^{2}$. We thus obtain:

$$
W_{l m n}=\frac{1}{8 \pi^{2}} \sqrt{\frac{2}{2 l+1}} C_{l}^{-n-m}
$$




\section{Comparison of the O.D.F. sections}

The $C_{l}^{m n}$ coefficients enable one to calculate numerical values of the function $f\left(\varphi_{1}, \Phi, \varphi_{2}\right)$. Because this function depends on three angular variables, it is usually represented as sections with one of the angles kept constant (Bunge, 1969, 1982).

As Euler angles used by Bunge and Roe differ, the plottings of the O.D.F. sections are generally not identical.

The two kinds of graphs can be deduced from one another by geometrical transformations which arise from the correspondence relations Eq. (1).

These geometrical transformations are particularly simple when the sample and the crystal lattice have symmetry elements. In the following we are going to describe these transformations in the major case of an orthorhombic sample symmetry and a cubic lattice symmetry.

As a matter of fact, the application of these symmetries allows us to restrict the variation range of all three Euler angles to the interval $\left[\mathrm{O}, \frac{\pi}{2}\right]$. Especially, for the Euler angles $\left\{\varphi_{1}, \Phi, \varphi_{2}\right\}$, the following equivalence relation holds:

$$
\left\{\varphi_{1}, \Phi, \varphi_{2}\right\} \equiv\left\{\pi-\varphi_{1}, \Phi,-\varphi_{2}\right\}
$$

The combination of the correspondence relations (1) and the symmetry relation (31) leads to:

$$
\begin{aligned}
\psi & =\frac{\pi}{2}-\varphi_{1} \\
\theta & =\Phi \\
\varphi & =\frac{\pi}{2}-\varphi_{2}
\end{aligned}
$$

These relations show that the $\psi$ and $\varphi_{1}$ variables are connected with one another by a symmetry plane at $\psi_{s}=\varphi_{1 s}=45^{\circ}$; likewise $\varphi_{s}=\varphi_{2 s}=45^{\circ}$. The plottings of the O.D.F. sections in the Euler space, in the terminology of respectively Bunge and Roe, correspond to one another by means of these symmetry planes, as shown in Figure 2.

\section{THE TWO DIMENSIONAL FUNCTIONS}

The mathematical relation which makes it possible to go from O.D.F. to pole figure is an integral (Bunge, 1969; Bunge, 1982). Thus the various functions necessary to study pole figures are only bi-dimensional. The 

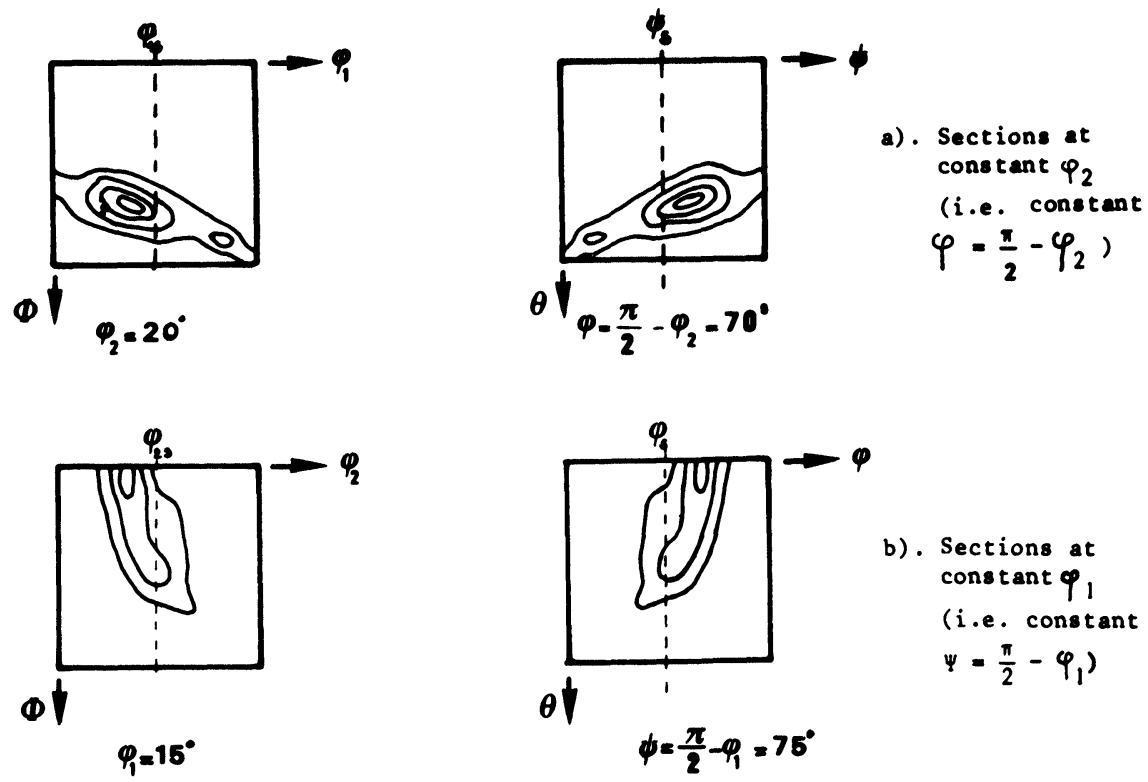

FIGURE 2 Correspondence between O.D.F. sections represented in the terminology of, respectively, Bunge and Roe

a) Sections at constant $\varphi_{2}$ (i.e. constant $\varphi=\frac{\pi}{2}-\varphi_{2}$ )

b) Sections at constant $\varphi_{1}$ (i.e. constant $\psi=\frac{\pi}{2}-\varphi_{1}$ ).

correspondence relations are then particular cases of the relations which have just been established for the three-dimensional functions.

\section{Spherical coordinates on the pole sphere}

The pole figure $P_{\mathbf{h}_{i}}$ is the distribution density of the normals $\mathbf{h}_{i}$ to a family of crystallographic planes $\left\{h_{i}, k_{i}, l_{i}\right\}$ in the sample reference frame.

The orientation of the normals to the considered planes is described with its polar coordinates. These polar coordinates make up an orthogonal curvilinear system of coordinates on the sphere; they are defined identically by both Bunge and Roe (Figure 3).

$$
\left\{\begin{array}{l}
\chi=\Phi \\
\eta=\gamma
\end{array}\right.
$$



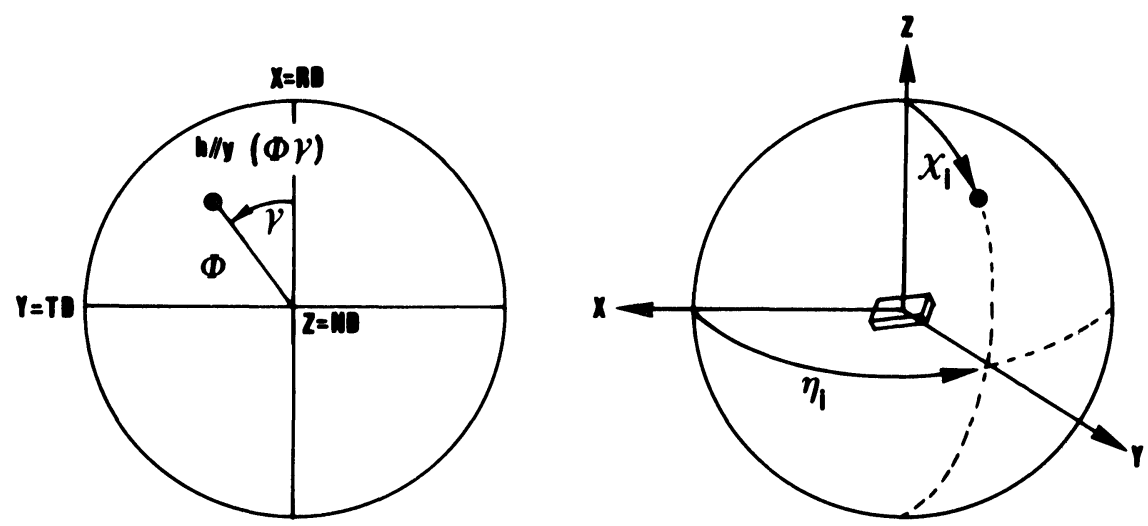

FIGURE 3 Coordinates on the pole sphere

a) Bunge's definition (Bunge, 1969; 1982)

b) Roe's definition (Roe, 1965).

\section{Relations between the polynomials $\boldsymbol{P}_{I}^{-m}(\cos \Phi)$ and $\boldsymbol{P}_{I}^{m}(\xi)$}

A pole figure can be developed into a series on the spherical surface harmonics. These functions constitute indeed a complete orthogonal basis on the sphere.

According to Bunge, this development reads:

$$
P_{\mathbf{h}_{i}}(\Phi, \gamma)=\sum_{l=0}^{\infty} \sum_{n=-l}^{+l} F_{l}^{n}\left(\mathbf{h}_{i}\right) \cdot k_{l}^{n}(\Phi, \gamma)
$$

with the following definition for the spherical surface harmonics:

$$
k_{l}^{n}(\Phi, \gamma)=\frac{1}{\sqrt{2 \pi}} P_{l}^{-n}(\Phi) e^{i n \gamma}
$$

In Roe's terminology the corresponding series expansion reads:

$$
q_{i}(\chi, \eta)=\sum_{l=0}^{\infty} \sum_{n=-l}^{+l} Q_{l n}^{i} P_{l}^{n}(\chi) e^{-i n \eta}
$$

The polynomials with three indices $l, m$ and $n$ are the polynomials associated to the Jacobi ones. When either index $m$ or $n$ is zero, we obtain the polynomials associated to the Legendre ones:

$$
P_{l}^{m}(\xi)=Z_{l m 0}(\xi)
$$

and

$$
\bar{P}_{l}^{m}(\xi)=i^{m} \sqrt{\frac{2 l+1}{2}} P_{l}^{m 0}(\xi)
$$


If we now take relation (28) into account together with the previous relations, the correspondence formula is immediately obtained:

$$
P_{l}^{m}(\xi)=(-1)^{m} \bar{P}_{l}^{m}(\xi)
$$

\section{Respective norms of the pole figures}

In Roe's notation the so-called plane-normal orientation distribution $q_{i}(\chi, \eta)$ is obtained by normalizing the diffracted intensity $I_{i}(\chi, \eta)$ :

$$
q_{i}(\chi, \eta)=I_{i}(\chi, \eta) / \int_{0}^{2 \pi} \int_{0}^{\pi} I_{i}(\chi, \eta) \sin \chi d \chi d \eta
$$

The pole figure is thus normalized to the unit according to the formula:

$$
\int_{0}^{2 \pi} \int_{0}^{\pi} q_{i}(\chi, \eta) \sin \chi \cdot d \eta \cdot d \chi=1
$$

In Bunge's notation, the measurement of the diffracted intensity $\hat{P}_{\mathrm{h}_{i}}(\Phi, \gamma)$ leads to the normalized pole figure $P_{\mathrm{h}_{i}}(\Phi, \gamma)$ using the relation:

$$
P_{\mathrm{h}_{i}}(\Phi, \gamma)=4 \pi \frac{\hat{P}_{\mathrm{h}_{i}}(\Phi, \gamma)}{\int_{0}^{2 \pi} \int_{0}^{\pi} \hat{P}_{\mathrm{h}_{i}}(\Phi, \gamma) \cdot \sin \Phi \cdot d \Phi \cdot d \gamma}
$$

Integrating the above relation, the following normalization condition is obtained:

$$
\int_{0}^{2 \pi} \int_{0}^{\pi} P_{\mathrm{h}_{i}}(\Phi, \gamma) \cdot \sin \Phi \cdot d \Phi \cdot d \gamma=4 \pi
$$

Therefore this normalization condition of pole figures differs from Roe's only by a multiplying factor of $4 \pi$ :

$$
q_{i}(\chi, \eta)=\frac{1}{4 \pi} P_{\mathrm{h}_{i}}(\Phi, \gamma)
$$

This relation is verified in the particular case of a random pole figure:

$$
q_{\text {rand. }}=\frac{1}{4 \pi} P_{\text {rand. }}
$$

The value of the constant $q_{\text {rand. }}$ is directly deduced from Eq. (41)

$$
q_{\text {rand. }} \int_{0}^{2 \pi} \int_{0}^{\pi} \sin \chi \cdot d \chi \cdot d \eta=1
$$


i.e.

$$
\left\{\begin{array}{l}
q_{\text {rand. }}=\frac{1}{4 \pi} \\
P_{\text {rand. }}=1
\end{array}\right.
$$

Just as for the function $f(g)(\S 3.2)$, this normalization determines the coefficient of the series when the degree $l$ is 0 .

$$
Q_{00}^{i}=\frac{\sqrt{2}}{4 \pi}
$$

and

$$
F_{0}^{0}\left(\mathbf{h}_{i}\right)=\sqrt{4 \pi}
$$

Relation between the coefficients $F_{l}^{n}\left(h_{i}\right)$ and $Q_{l n}^{i}$ of the pole figures

If in Bunge's expression of the pole figure the angles $(\Phi, \gamma)$ are replaced by Roe's, i.e. $(\chi, \eta)$, the relation (44) becomes:

$$
q_{i}(\chi, \eta)=\frac{1}{4 \pi} \sum_{l=0}^{\infty} \sum_{n=-l}^{+l} F_{l}^{n}\left(\mathbf{h}_{i}\right) \cdot \frac{1}{\sqrt{2 \pi}} \bar{P}_{l}^{n}(\chi) \cdot e^{i n \eta}
$$

Thus:

$$
q_{i}(\chi, \eta)=\sum_{l=0}^{\infty} \sum_{n=-l}^{+l} \mid \frac{1}{4 \pi \sqrt{2 \pi}} F_{l}^{-n}\left(\mathbf{h}_{i}\right)^{l} P_{l}^{n}(\chi) \cdot e^{-i n \eta}
$$

taking the relations Eq. (39) and

$$
\bar{P}_{l}^{-n}(\chi)=(-1)^{n} \bar{P}_{l}^{n}(\chi)
$$

into account.

Equation (51) makes the relationship between the respective coefficients conspicuous:

$$
Q_{l}^{i}=\frac{1}{4 \pi \sqrt{2 \pi}} F_{l}^{-n}\left(\mathbf{h}_{i}\right)
$$

The coefficients (Eq. 48-49) obviously verify the above relation in the specific case when $l=0$. 


\section{CONSIDERING THE SYMMETRIES OF THE TEXTURE FUNCTION}

\section{The dual origin of symmetries: crystal and sample symmetry}

The $f(g)$ texture function satisfies two invariance types that result from the crystal symmetry on the one hand, from the sample symmetry on the other hand. The symmetries of a crystal lattice can be defined by a symmetry point group where $G_{B}$ is the rotation subgroup:

$$
G_{B}=\left\{g_{j}^{B}\right\}_{j=1, N_{B}}
$$

Due to these symmetries, the $f(g)$ function is invariant for the corresponding orientations which could not physically be distinguished from one another:

$$
f\left(g_{j}^{B} \cdot g\right)=f(g)
$$

In the same way the crystallites distribution in the polycrystal may have statistical symmetries $g_{i}^{A}$ which generally are induced by the shaping processes of the material:

$$
f\left(g \cdot g_{i}^{A}\right)=f(g)
$$

Both invariance conditions Eq. (55-56) can be taken into account independently from one another; indeed one of them operates on the right whereas the other one operates on the left. The treatment of these symmetries being otherwise identical, only either type is to be examined.

Bunge creates functions adapted to the symmetries by means of linear combinations of the generalized spherical harmonics $T_{l}^{m n}(g)$ (Bunge, 1965b; 1982). Roe expresses the $W_{l m n}$ coefficients of the series as being a function of only a smaller number of these, which are linearly independent (Roe, 1966).

\section{The "lower" symmetry (non-cubic): selection rules}

We will take the trigonal crystal symmetry and the orthorhombic sample symmetry as examples and investigate these two cases.

The trigonal crystal symmetry Let us recall that the rotations subgroup of the trigonal holohedrism is characterized by one threefold axis and perpendicular to it, three binary axes. The threefold axis (parallel to the $Z$ axis of the $K_{B}$ system) selects the generalized spherical harmonics $T_{l}^{3 m, n}(g)$ whose left index is a multiple of 3 . Likewise it selects the $W_{l m 3 n}$, the other coefficients being necessarily zero.

Moreover a binary axis operates a grouping of functions whose $3 \mathrm{~m}$ indices 
have opposite signs, providing an orthonormal symmetrized basis:

$$
\begin{aligned}
& \dot{T}_{l}^{1 n}(g)=\frac{1}{2}\left[1+(-1)^{l}\right] T_{l}^{0 n}(g) \\
& \vdots \\
& \dot{T}_{l}^{\mu n}(g)=\frac{1}{\sqrt{2}}\left[T_{l}^{3 m n}(g)+(-1)^{l+m} T_{l}^{-3 m n}(g)\right], \mu>1
\end{aligned}
$$

with

$$
3 m=3(\mu-1)
$$

For similar reasons. the series coefficients according to Roe verify the following linear dependence relation:

$$
W_{l m 3 n}=(-1)^{l+n} W_{l m-3 n}
$$

The orthorhombic sample symmetry The rotations subgroup is characterized by three binary axes perpendicular two by two. The description of the symmetry conditions is simple when the sample coordinate system $K_{A}$ is parallel to these binary axes (Figure 4).

As in the above case the binary axis parallel to the $Z$ axis selects the corresponding even indices, whereas a binary axis perpendicular to $X$ gathers the even indices with opposite signs.

In Bunge's procedure, the new orthonormal basis reads:
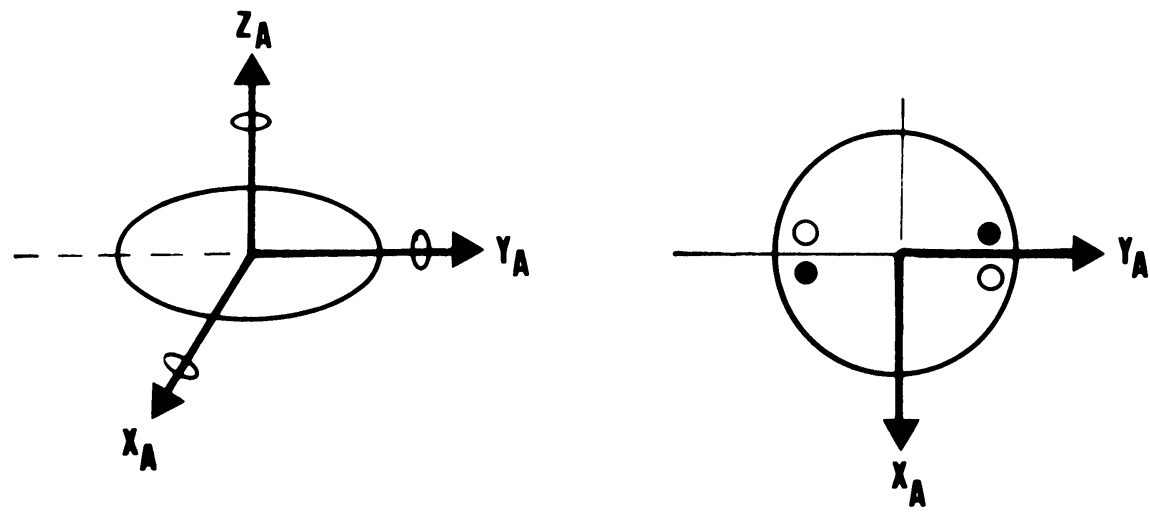

FIGURE 4 Setting of the $K_{A}$ coordinate system with respect to the orthorhombic binary axes. 


$$
\begin{aligned}
& \dot{T}_{l}^{m 1}(g)=\frac{1}{2}\left[1+(-1)^{l}\right] T_{l}^{m 0}(g) \\
& \dot{T}_{l}^{m v}(g)=\frac{1}{\sqrt{2}}\left[T_{l}^{m 2 n}(g)+(-1)^{l} T_{l}^{m-2 n}(g)\right], v>1
\end{aligned}
$$

with

$$
2 n=2(v-1)
$$

Similarly, according to Roe, the series coefficients whose $m$ indices are necessarily even, are moreover bound by the following condition:

$$
W_{l 2 m n}=(-1)^{l} W_{l-2 m n}
$$

\section{The cubic symmetry case: symmetry coefficients according to Bunge and Roe}

The description of the symmetries is simplified if the $Z$ axis of the crystal system is parallel to the four-fold axis which has the highest multiplicity. Considering moreover a binary axis orthogonal to the four-fold axis implies describing the tetragonal symmetry which corresponds to a subgroup of the cubic symmetry.

In Bunge's view the functions adapted to the tetragonal symmetry are:

$$
\begin{aligned}
& \dot{T}_{l}^{1 n}(g)=\frac{1}{2}\left[1+(-1)^{l}\right] T_{l}^{0 n}(g) \\
& \dot{T}_{l}^{\mu n}(g)=\frac{1}{\sqrt{2}}\left[T_{l}^{4 m n}(g)+(-1)^{l} T_{l}^{-4 m n}(g)\right], \mu>1
\end{aligned}
$$

with

$$
4 m=4(\mu-1)
$$

Parallel to this the only non-zero coefficients $W_{l m 4 n}$ in Roe's series also verify:

$$
W_{l m 4 n}=(-1)^{l} W_{l m-4 n}
$$

Equations (63-65) do not take into account the threefold axis which is at an angle of $54^{\circ} 44^{\prime}$ with the four-fold axis.

Due to the particular value of this angle, the functions adapted to the cubic symmetry cannot be obtained as above using only selection rules. The functions adapted to the cubic symmetry are linear combinations of spherical harmonics:

$$
\dot{T}_{l}^{\mu n}(g)=\sum_{4 m=-l}^{+l} \dot{A}_{l}^{4 m \mu} T_{l}^{4 m n}(g)
$$


As the real $\dot{A}_{l}^{4 m \mu}$ coefficients verify the relation:

$$
\dot{A}_{l}^{-4 m \mu}=(-1)^{l} \dot{A}_{l}^{4 m \mu}
$$

the above mentioned linear combination also reads:

$$
\dot{T}_{l}^{\mu n}(g)=\dot{\dot{A}}_{l}^{0 \mu} T_{l}^{0 n}(g)+\sum_{4 m=4}^{l} \dot{\dot{A}}_{l}^{4 m \mu}\left[T_{l}^{4 m n}(g)+(-1)^{l} T_{l}^{-4 m n}(g)\right]
$$

The $\mu$ index takes on the values $1,2,3 \ldots M(l)$ and so numbers the elements of the orthogonal basis:

$$
\int \dot{T}_{l}^{\mu n}(g) \dot{T}_{l^{\prime}}^{* \mu^{\prime} n^{\prime}}(g) d g=\frac{1}{2 l+1} \delta_{l l^{\prime}} \delta_{\mu \mu^{\prime}} \delta_{n n^{\prime}}
$$

In the case of lower symmetry, the number of functions adapted to the symmetries is obtained simply by counting from their definition. Thus in the case of the tetragonal symmetry, the number of functions is an immediate result of the selection rules expressed in Eq. (63-64):

$$
\begin{array}{ll}
{\left[\frac{l}{4}\right]+1} & l \text { even } \\
{\left[\frac{l}{4}\right]} & \text { lodd }
\end{array}
$$

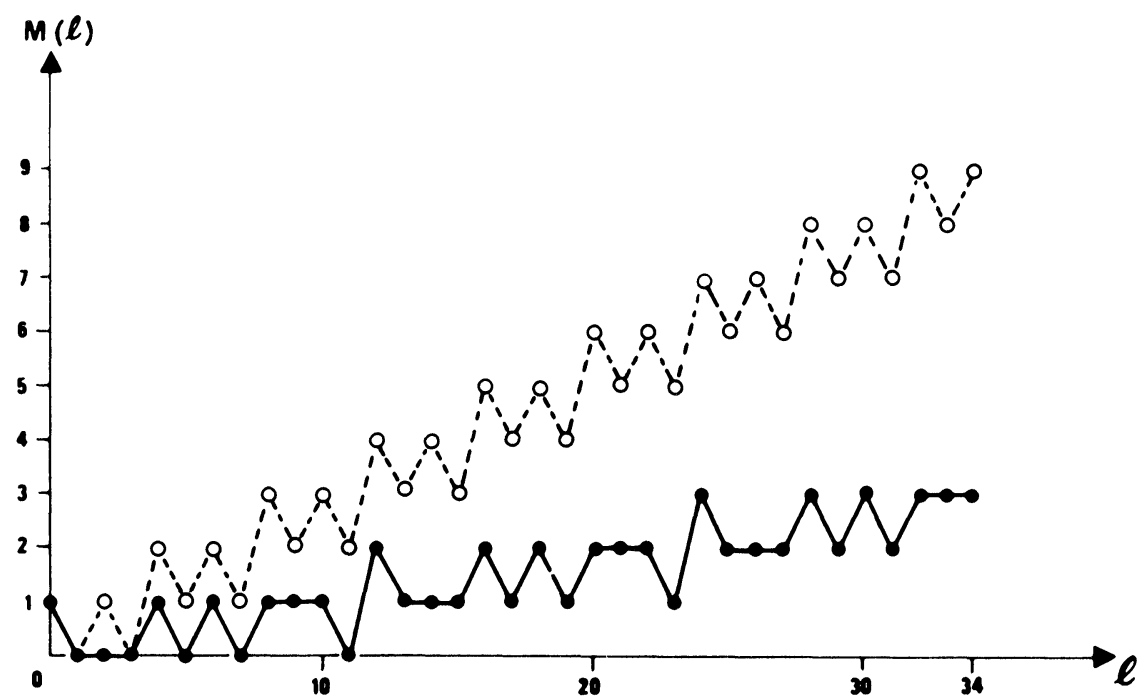

FIGURE $5 \quad M(l)$ number of linear independent symmetry-adapted functions corresponding to tetragonal symmetry (dotted curve) and cubic symmetry (solid curve). 
where the square brackets symbolize the integral part of the division. The $M(l)$ number of cubic harmonics is noticeably lower than the number (Eq. 70) of functions of tetragonal symmetry (Figure 5).

The calculation of $M(l)$ does not result only from a counting, but it requires using the group theory (Esling, 1981; Bechler-Ferry, 1981). The $\dot{A}_{l}^{4 m \mu}$ are often used in a somewhat different type of normalization:

$$
\begin{aligned}
& \dot{B}_{l}^{0 \mu}=\dot{A}_{l}^{0 \mu} \frac{1}{\sqrt{2 \pi}} \\
& \dot{B}_{l}^{4 m \mu}=\dot{A}_{l}^{4 m \mu} \frac{2}{\sqrt{2 \pi}}
\end{aligned}
$$

These $\dot{B}_{l}^{4 m \mu}$ coefficients more specifically lead to a concise expression of the spherical surface harmonics (Bunge, 1969; 1982):

$$
\dot{k}_{l}^{\mu}(\Phi, \beta)=\sum_{4 m=0}^{l} \dot{B}_{l}^{4 m \mu} \bar{P}_{l}^{4 m}(\Phi) \cos 4 m \beta
$$

They are tabulated for all the integer values of $l$, up to the degree $l=34$ (Table I).

$M(l)$ is also the number of linearly independent $W_{l m 4 n}$ coefficients. Whenever $l$ is odd the first coefficient corresponding to $4 n=0$ is necessarily zero:

$$
W_{l m 0}=0
$$

Just as Roe did, we shall express the $W_{l m 4 n}$ texture coefficients in relation to the $M(l)$ coefficients occurring first, these being selected as independent from one another.

These relations read:

$$
W_{l m 4 n}=\sum_{\mu=1}^{M(l)} R_{4 n \mu}^{l} W_{l m 4(\mu-1)}
$$

when $l$ is even; taking Eq. (73) into account:

$$
W_{l m 4 n}=\sum_{\mu=1}^{M(l)} R_{4 n \mu}^{l} W_{l m 4 \mu}
$$

when $l$ is odd.

In his paper (Roe, 1966) Roe submitted these relations for even values of $l$ smaller than 22. Table II shows the extension of Roe's $R_{4 n \mu}^{l}$ symmetry coefficients to all the $l$ integer values up to 34 .

\section{Double symmetrized functions}

The texture function is usually developed into series 


$$
f(g)=\sum_{l \mu v} C_{l}^{\mu v}:_{T_{l}^{\mu v}}(g)
$$

on the basis of spherical harmonics that verify the double invariance:

$$
\check{T}_{l}^{\mu \nu}\left(g_{j}^{B} \cdot g \cdot g_{i}^{A}\right)=\check{T}_{l}^{\mu \nu}(g)
$$

As has been seen above, either symmetry is imposed independently from one another. Rolled metal sheets usually show an orthorhombic sample symmetry. But on the other hand geological samples generally do not offer any statistical symmetry (triclinic symmetry).

On the other hand the texture transformation function has been introduced by Bunge (1969) for recrystallization:

$$
W(\Delta g)=\sum_{l \mu \nu} C_{l}^{\mu \nu} \ddot{T}_{l}^{\mu \nu}(\Delta g)
$$

This function makes it possible to describe not only recrystallization but also texture transformations by phase change. In the latter case the symmetries of the initial phase operate on the right and those of the resulting phase operate on the left. Functions of cubic-cubic symmetry are therefore required in the very important case of martensitic transformation.

What we have said in the above paragraph can easily be translated into Roe's terminology, we leave it up to the reader to do this transposition for himself.

\section{Note}

The reader will profitably refer to section 11 "Comparison between the terminologies of Bunge and Roe" in the book Quantitative Texture Analysis. This paper, which is written in a didactic form and compact style, avoids as many developments based on pure mathematical procedures as possible. It nevertheless contains all the information and relations required for the comparison of scientific results obtained with Bunge's or Roe's methods.

\section{References}

Bechler-Ferry, E. Contribution au Développement de l'Analyse des Textures. Extension aux Rangs Impairs, Thesis, University of Metz (1981).

Bunge, H. J. Z. Metallkde. 56, 872 (1965).

Bunge, H. J. Mber., Dt. Akad. Wiss. 7, 351 (1965b).

Bunge, H. J. Mathematische Methoden der Texturanalyse, Akademie Verlag, Berlin (1969).

Bunge, H. J. Textures in Material Science Mathematical Methods. Butterworth, London, in press (1982).

Bunge, H. J. and Esling, C. Quantitative Texture Analysis. Deutsche Gesellschaft für Metallkunde, Société Française de Métallurgie, Frankfort, Paris (1982).

Esling, C. Effets des Symétries des Cristaux, des Echantillons et de la Diffraction sur la Définition et la Determination de la Fonction de Texture, Thesis, University of Metz (1981).

Roe, R. J. J. Appl. Phys. 36, 2024 (1965).

Roe, R. J. J. Appl. Phys. 37, 2069 (1966). 


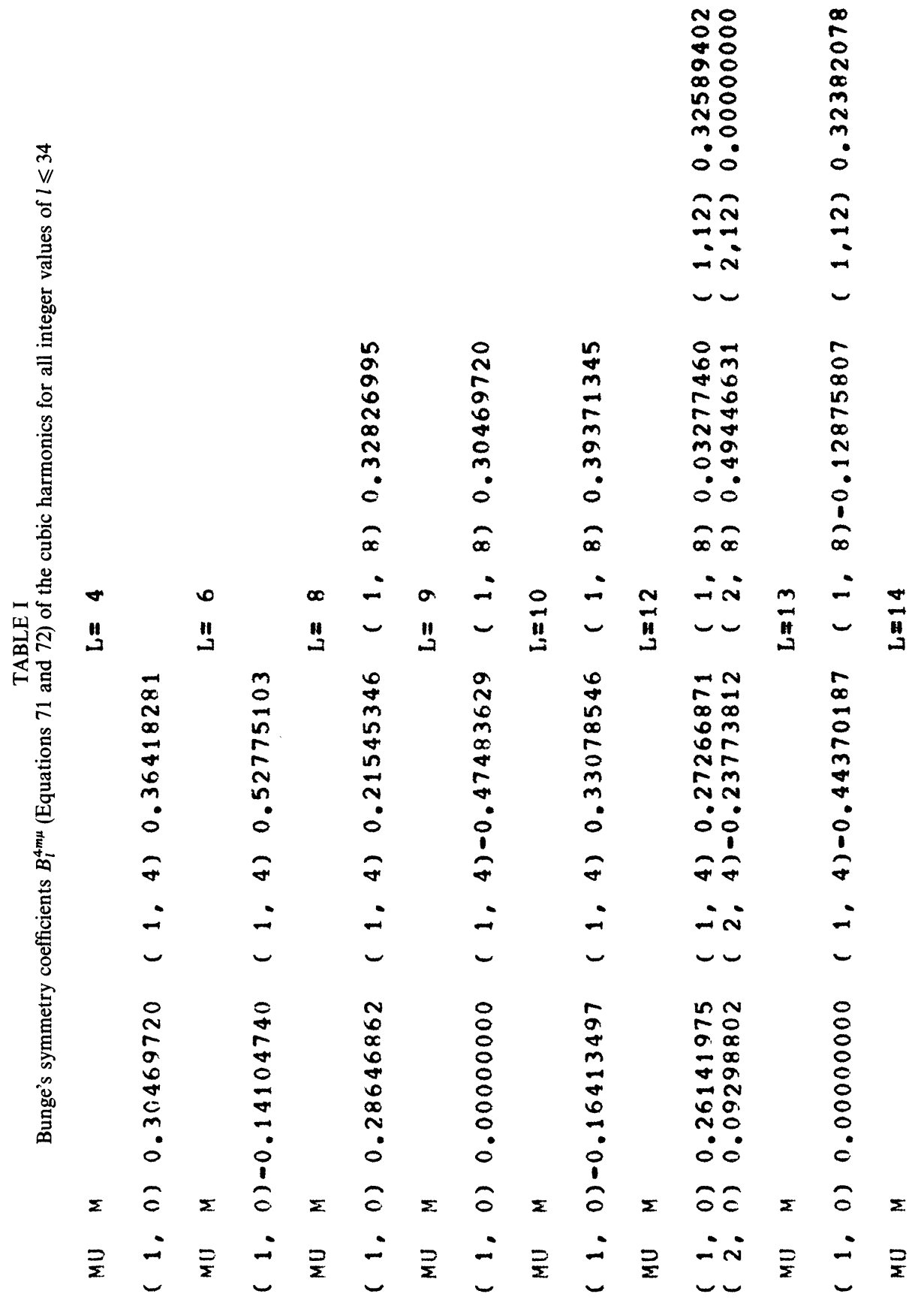



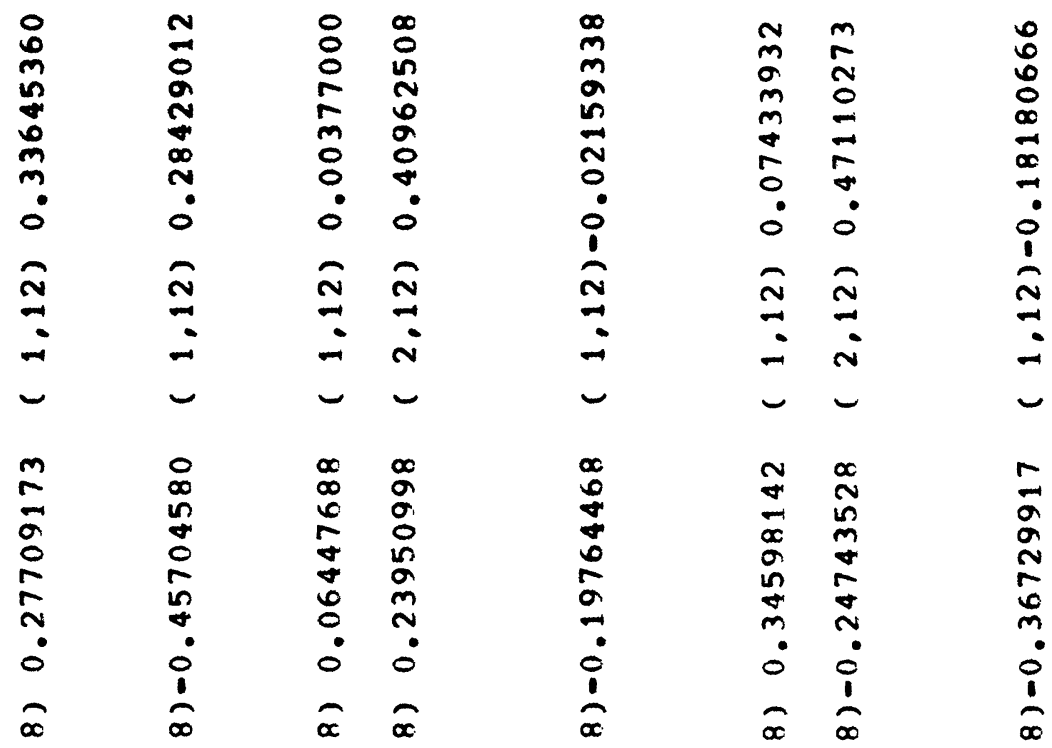

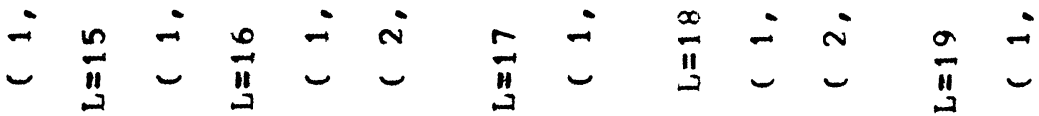
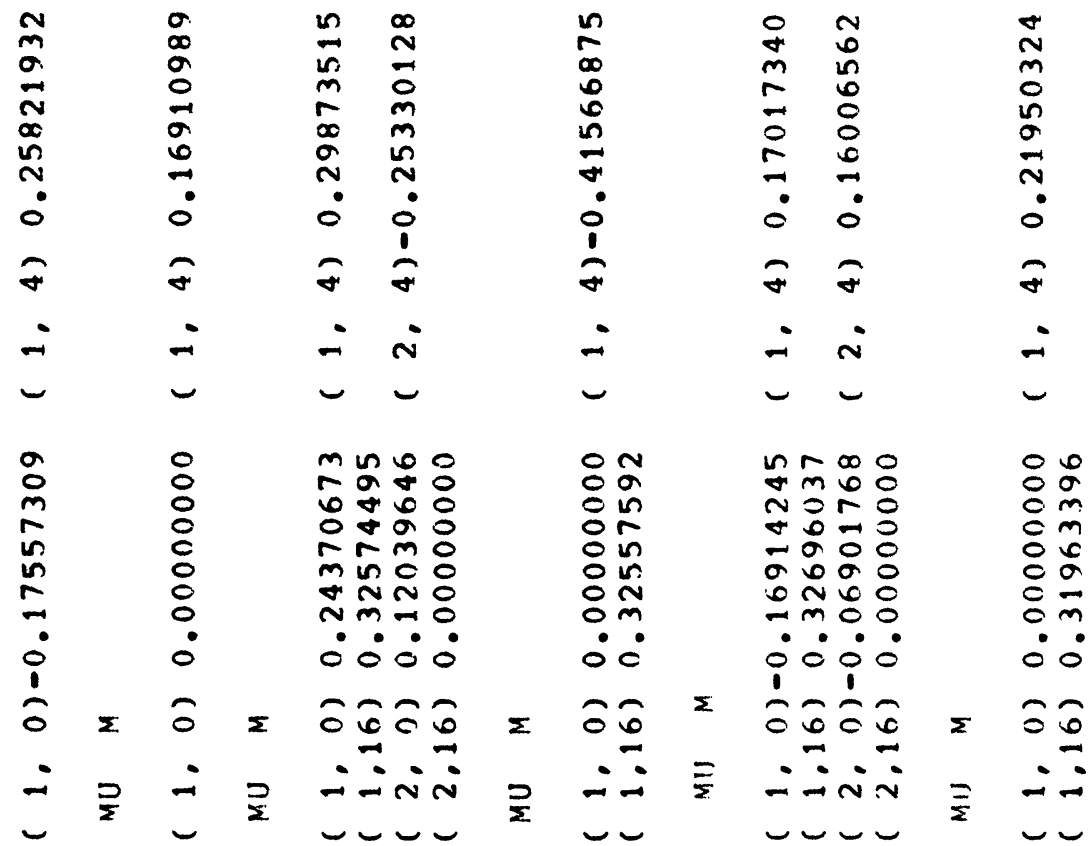

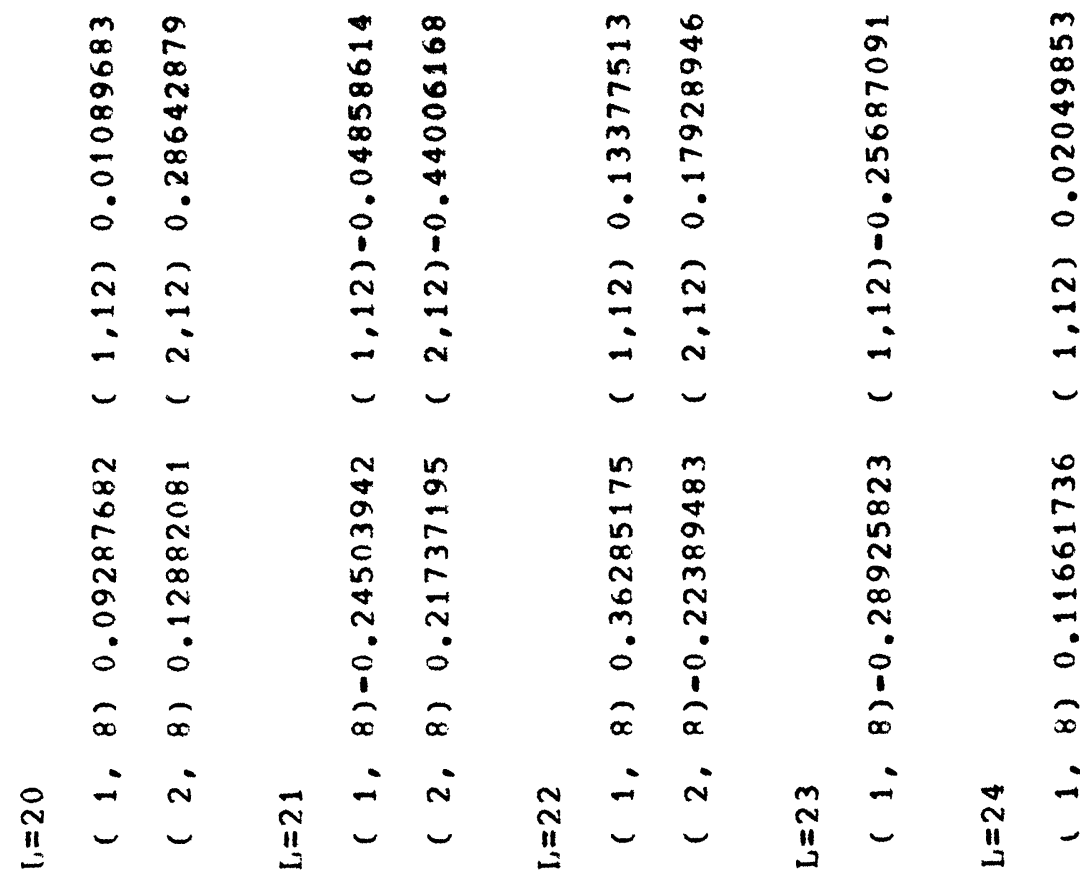

$m$
$n$
$\infty$
0
0
0
0
0
0
-1
-1

Nm 0

$\infty$ n 00

$m m \infty$

n

- in 00

$-N \operatorname{in} 0$

$m m N O$

$\circ: \circ 0^{\circ}$

000

12

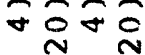

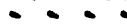

$\begin{array}{ll}\sim & n \\ a & n \\ m & n \\ n & n \\ \sim & n \\ \vdots & 0 \\ \infty & \infty\end{array}$

$\begin{array}{ll}n & m \\ n & \infty \\ n & 0 \\ \infty & \infty \\ N & m \\ 0 & N \\ m & \sim \\ 0 & 0 \\ 0 & 0\end{array}$

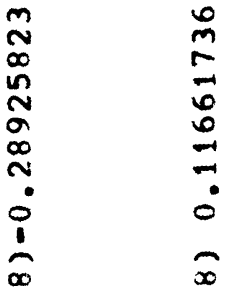

$-1 N \sim$

$\stackrel{i}{i}-\dot{v}$

$\stackrel{N}{N}=\dot{N}$

$\stackrel{-}{11}-$

$\infty m \backsim 0$

$m$ in $\propto$

N $N 0$

$\sim N-N$

$0 r m$ in

$\infty N \infty$ ?

$m m 00$

- 0 :

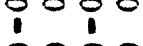

$\infty \infty-0$

+ un $\infty$

$+\infty \forall 0$

N $N$ N

- NO

-!?

$\because 0 \div 0$

$+0_{N}+0$

$-i N N$

0000

$\stackrel{m}{n}$

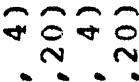

- -1

N n

in 6

$\theta r$

No

$\pi \pi$

n

i०

$+2$

$\smile \smile \smile \smile$

$\smile \smile \smile$
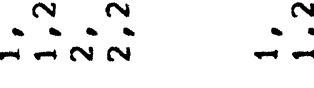

$n$
0
0
5
$m$
0
0
-

$v \& \circ m$

No $N$

$0 \ln \pi$

rano

c $m-n$

0010

Nom

: $: \circ 0$

$0+0 m$

0 in 0 in

$0 \mathrm{NO}$

$0 \propto 0-$

- Non

0006

- $0 \mathrm{~N}$

- 0.0

$\smile \smile \smile \smile$

$\smile \smile$

ㅇำ

n $m$ ON

a $\rightarrow 0 N$

$0-\pi$

- m 0

$-00 \%$

:0:0ं

000

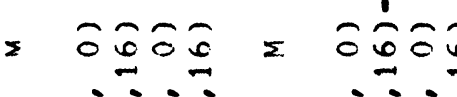

$=2 \frac{1}{0} 010$

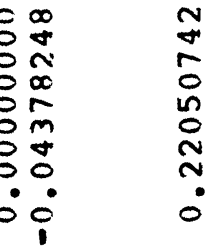

$\equiv-i N N$

$\geqslant-\pi N$

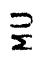

iñ

$00 \leq 0$

$\therefore$

군 


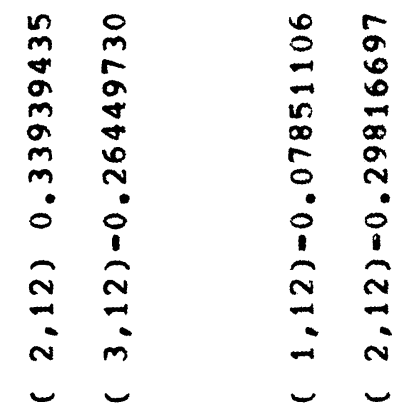

n 0 \% ก 00 N mNONO NNONO ก n 0 No NO 0.00 mo $0=0$ - $: 0 \circ 0^{\circ}$ $\cap \pi \cap \infty$ $\mathrm{N} N$ - v r m $\sim \sim \sim \sim$

cono 0 * 0 or $\infty 0$ $m \infty \approx n 0$ $0 \infty m+0$ 0 m a o 0 $0-N-0$ $0 N m-0$ - $0^{\circ} 0^{\circ} \circ$ 10 กิก ก $N$ N - $v$ rim $\sim \smile \smile \smile$

$-\pi N 00$ - Nora in $\infty \pi m$ in $\sigma$ $0-r \infty m$ n $n m R N$ $\rightarrow 10 \mathrm{~m}$ o $0 \mathrm{~m} 0 \mathrm{in}$ $\because \because \div . \div$ $\therefore \because 000$ ลิองอิ

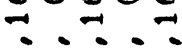
$\operatorname{rinmm}$ on $\infty$ or 0 - $0=0$ $\rightarrow+0$

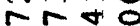
in $\cos 0$ $r N$ un 0 N $N 0$

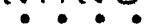
0000 1 กา 2 $\propto \sim \infty \propto$ $N N$ $\stackrel{n}{n-\pi n+\infty}$ II

$-\infty 00$ *ron on $r-\ln \infty$ $* m \sigma 0$ $00 \mathrm{rm}$ - $0 N-$ $m 0 m$ - 0 : 1.1

ก $2 \pi$

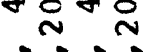

$-\infty N$

$\sim \omega \sim$

$\begin{array}{llll}0 & \cdots & 0 & 0 \\ 0 & 0 & 0 & 0\end{array}$

0 in 0

○ $0 \mathrm{om}$

0

C0ㅇ

$000 \mathrm{~N}$ $\therefore \therefore \circ:$ 101

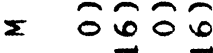

$\because \because 3$

ए

- in

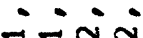

$\sim \sim \omega$

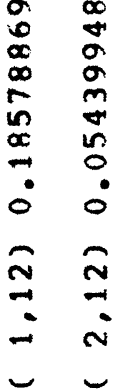

$\infty \pi n$ $\infty 0$ $+\infty 60$ NROO in in $r$ in $N \infty 0$ $m m=0$<smiles>CCCC</smiles>
0000 2) 2

$\infty+\infty$

$-2$

i $\because$ -

$\sim \sim \sim$

$0 n-m$

$m$ o $\infty$ n

$\omega-\infty \mathrm{m}$

$-10 N$

$0 \infty \mathrm{m}$

$\sim-0$

in $0 \mathrm{~N}$ in

- $0 \mathrm{Nm}$

:0ं0ं

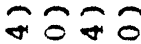

a

$-i N$

$\smile \smile \smile \smile$

$m \tilde{0}=0$

0 n 0

in $x$ N

$\underset{\sim}{\sim} \underset{m}{N}$

$n m-\infty$

m $-N$

:0:0:

0

1

กิ

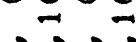

- -nn

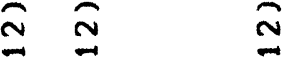

$-\infty$

$-$

$-\sim$

$\pi+20$

ANO

on $\Omega 00$

$\propto$ o

$m$ in 00

N $N$

- $\circ$

0000

$\stackrel{2}{2}$

$\infty \pi x$

N $N$

- $-i n i \infty$

II

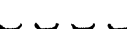

$\stackrel{\sim}{\sim} \sim$

$\sim m m+$

$\sim \pi \infty m$

$0 \sim N \infty$

$0 \infty \infty \sigma$

$0 r \infty 0$

c. 0 \&

NOON

:0:0ं 1

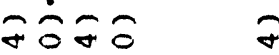

N N

$-\sim \sim N$

$\underbrace{\infty}_{0}$

2

$\smile \smile \smile \smile$

$\smile \smile \smile \smile \smile$

$\smile \smile \smile$

$0 N=0$

$0 \sim 0 \%$

- 250

$0 \infty 0 \sigma$

둥

$000 \%$

- $0 \circ 0$

$0 \% ?$

0

$n$

$m$

N

$m$

0

8

$-$

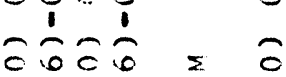

$\smile \smile \smile \smile$

$\overline{5}$

- $-?$

$\equiv$ 


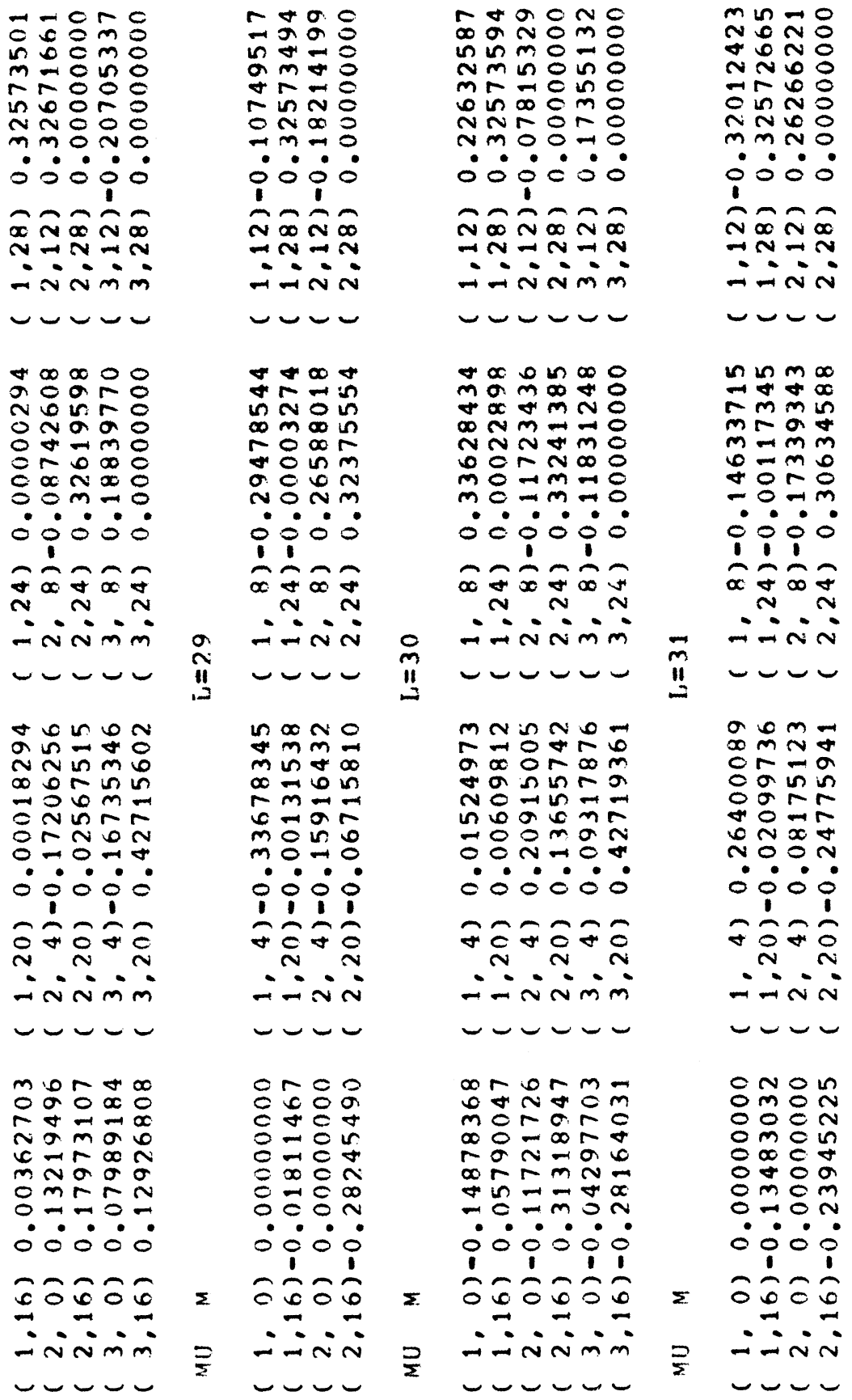




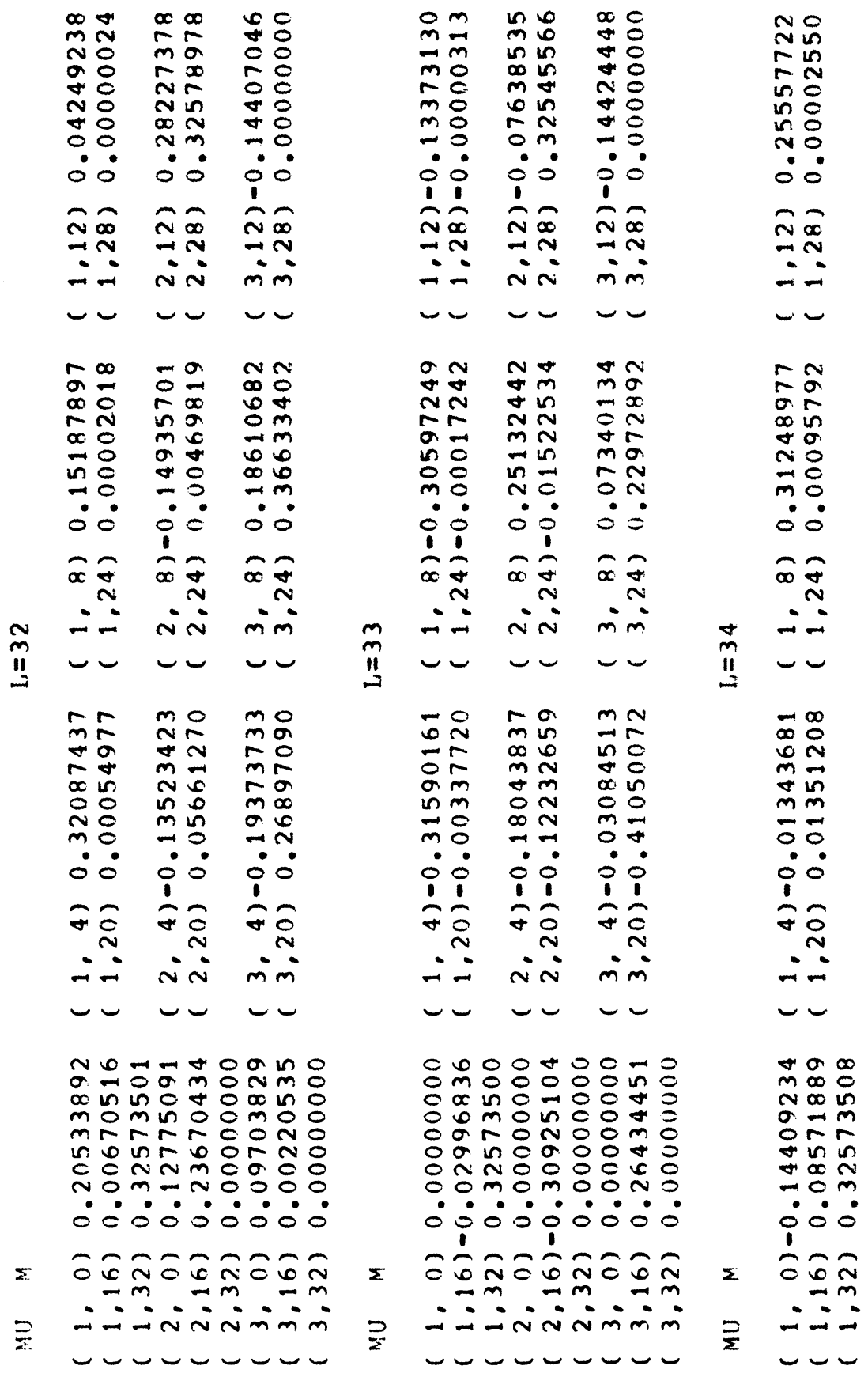




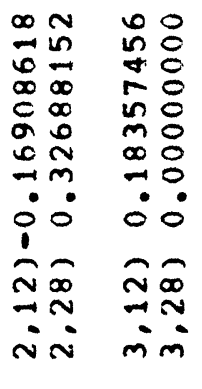

$\smile \smile \smile \smile$

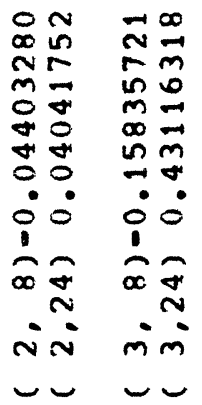

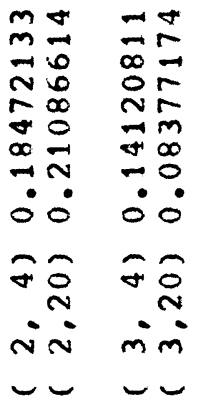

mก

$x 00 m 60$

r

nNorno

in Normo

$\rightarrow \pi 0000$

. 000000

ดิธกิธก

$\rightarrow m \rightarrow m$

Nivimin 


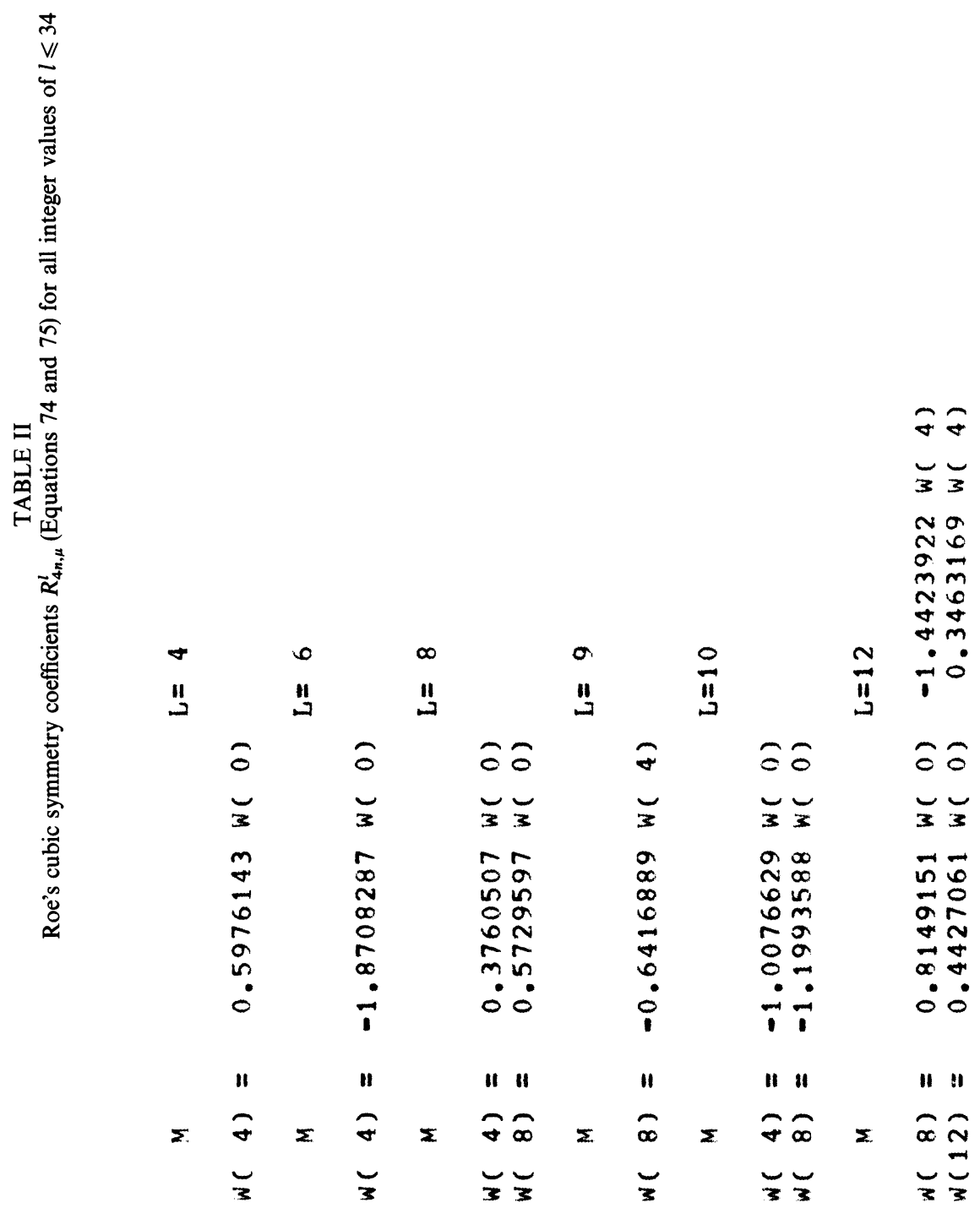




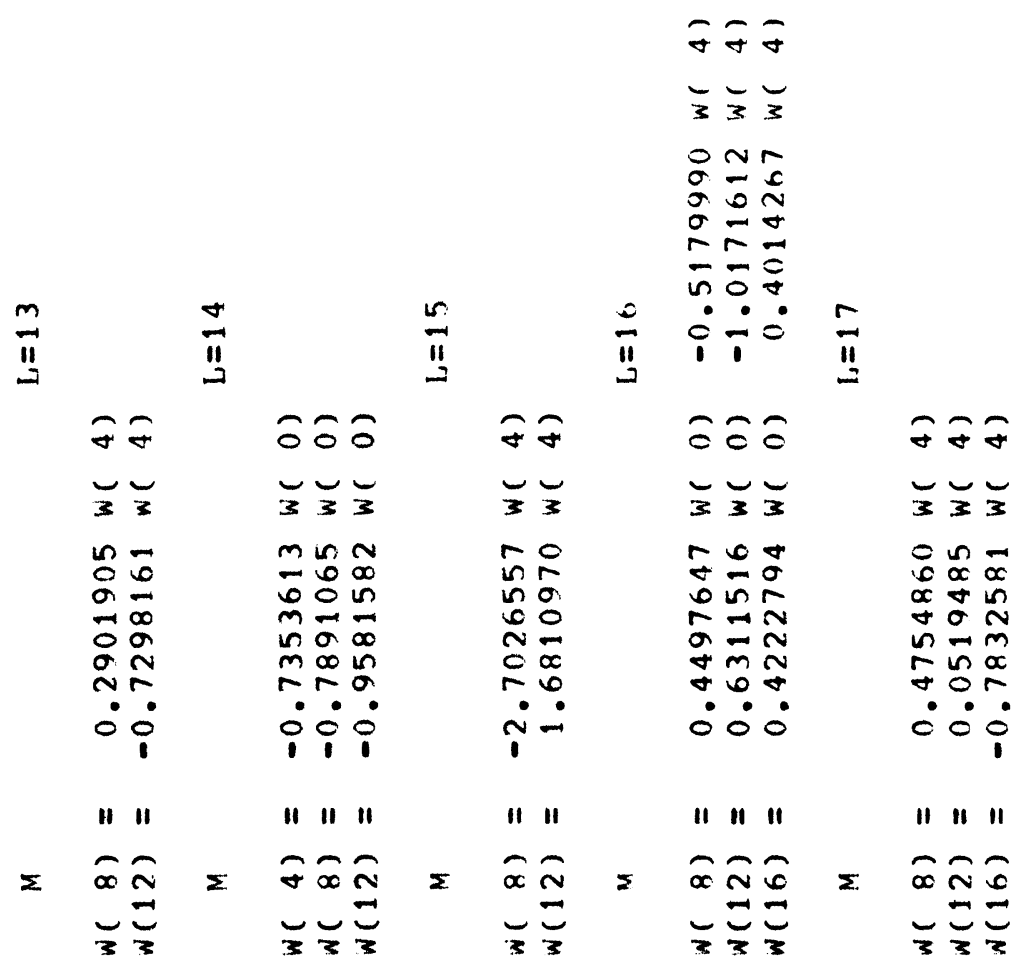




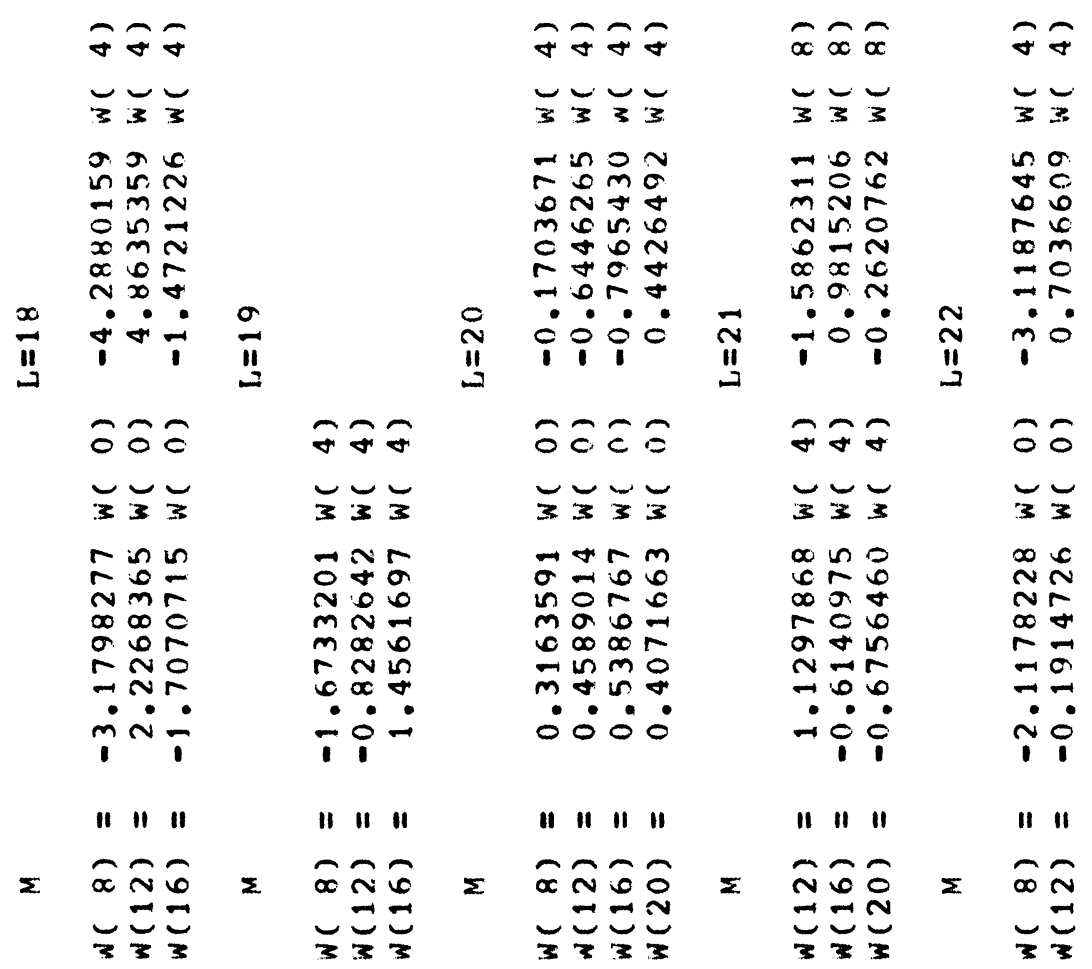




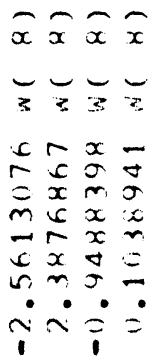

$\approx$

$\overparen{F}$
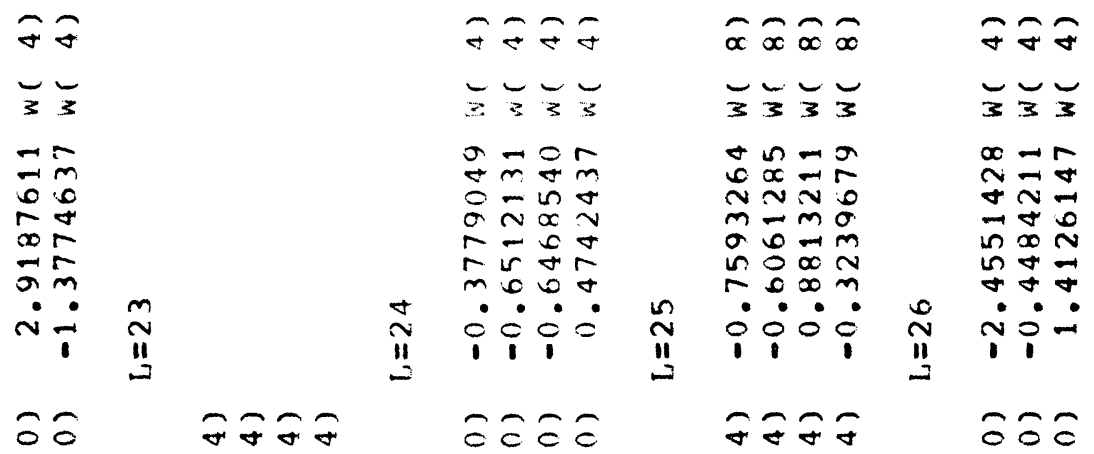

는

$\because \bar{m} \approx n$

$0-\cos$

$a \sim \propto \sim$

$r-\pi)$

$r$ ir $4 r$

नin

누 $\stackrel{m}{11}$

$m_{0} 0 \pi$

: 100

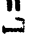

$2 \partial 2$

$6 \%$

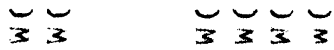

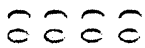

$\smile \smile \smile \smile$

$\smile \smile \smile$

× 3 उ 3

3333

333

$m \infty$

$\stackrel{m}{m} \underset{m}{m}$

$0 \sim \infty \infty$

$0-\infty-$

$\propto \bar{N} \bar{n}$

$\sin \theta 0$

듬

-

$\bar{\sim} \stackrel{\sim}{\sim} \stackrel{\infty}{\infty}$

$\sigma \alpha 0 m$

$r \sigma$

a

$x+$

$0 \infty \sigma \forall$

$0 \times v \sigma$

onㅔ

$\because 0 \div m$

$\div \div \div \div$

$\therefore \dot{0}: \dot{0}$

in o N $x$

$x \propto m n$

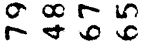

- $\bullet$

$000 \%$

\&ñ

$\sim N \frac{\pi}{2}$

o $\infty$ in

or $m$

in $4 \div$

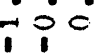

I "

" " | "

" | " 1"

" | | "

" " 11

कo $\Sigma$ क

$\Sigma$

วิ

$\mathbf{z}$

กิธีั

กิธิธ

-

$33=3$

כこบ

$\because \underset{3}{3}$ 

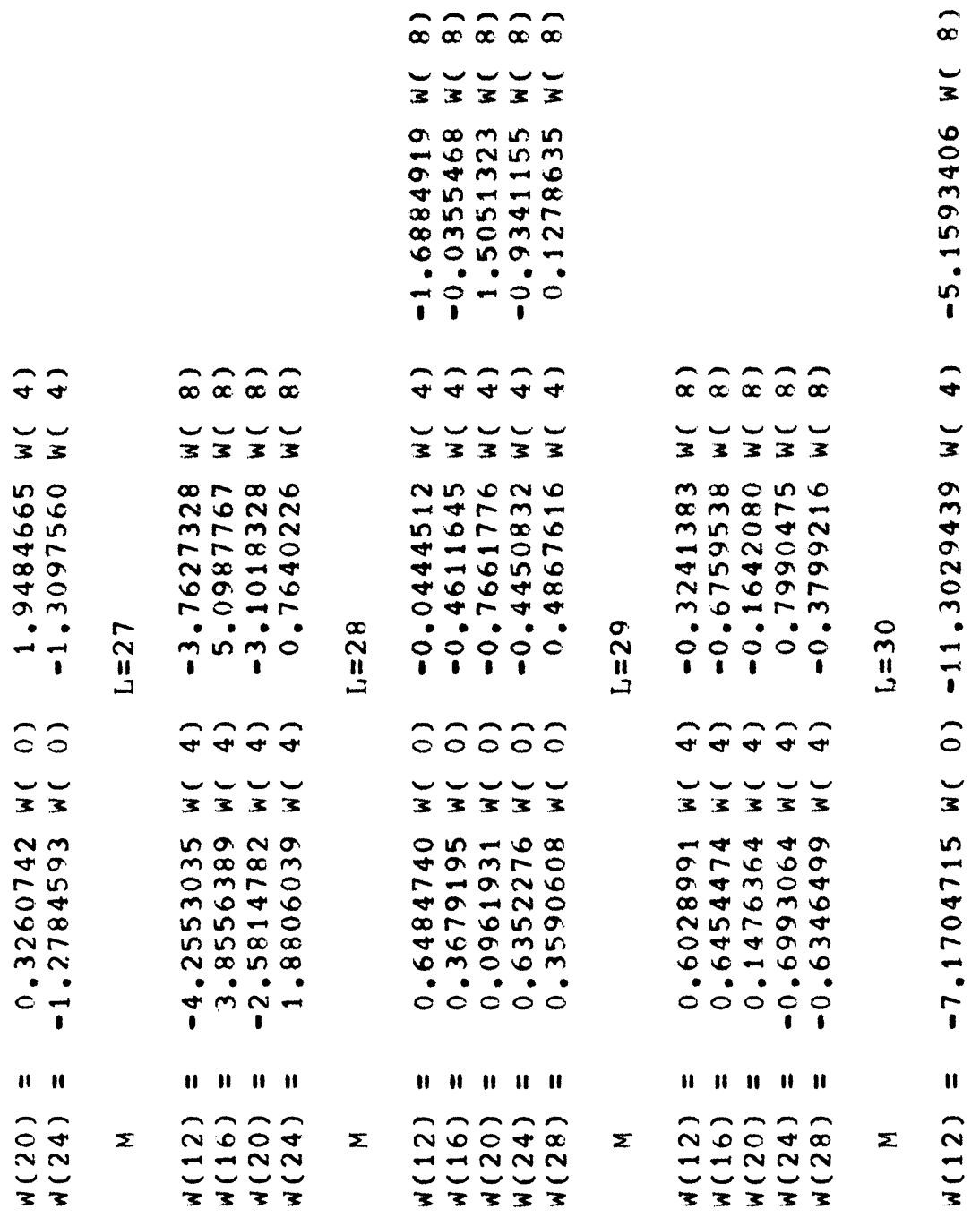

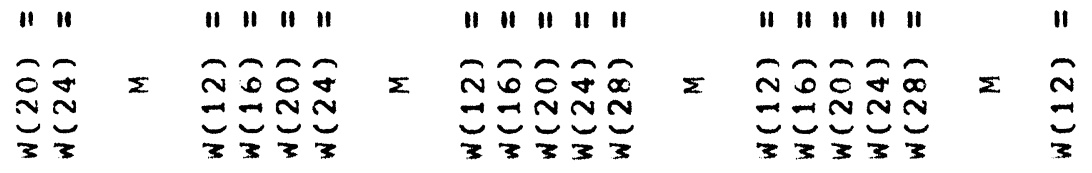




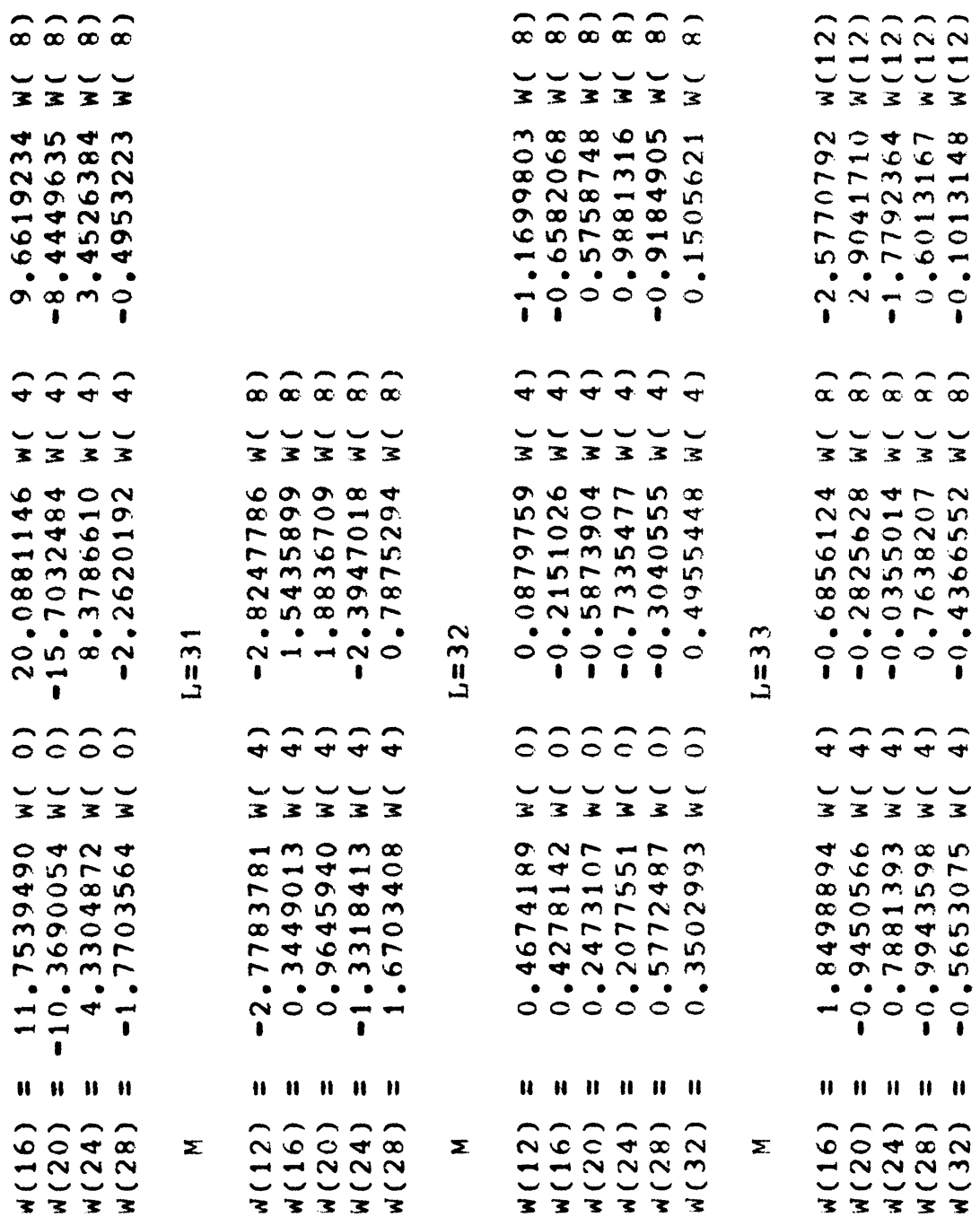




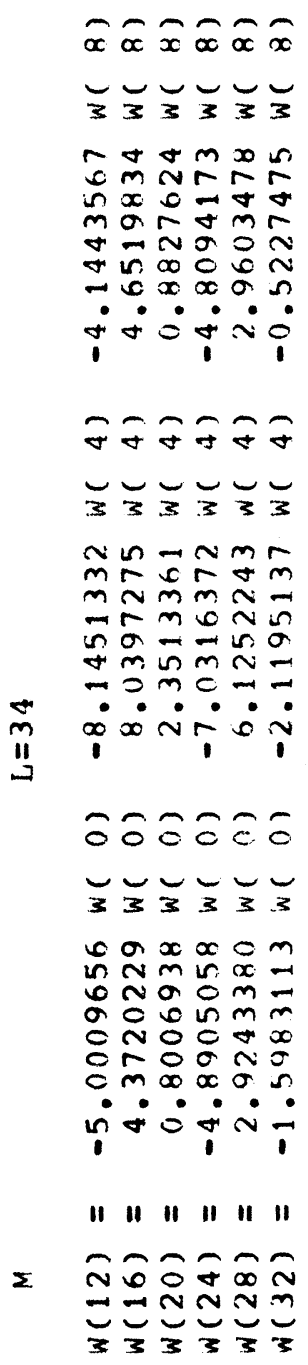

